\title{
Angiography and Therapeutic Embolisation of Bleeding Aberrant lliac Artery Branches
}

\author{
Muhammad Azeemuddin1, Misha Khalid Khan², Muhammad Danish Barakzai1, Muhammad Ghazi Asad Khan³, \\ Raza Sayani1 and Dawar Burhan Ahmed Khan ${ }^{1}$ \\ IDepartment of Radiology, The Aga Khan University Hospital, Karachi, Pakistan \\ 2The Aga Khan University Medical College, Karachi, Pakistan \\ ${ }^{3}$ Liaquat University of Medical and Health Sciences, Jamshoro, Sindh, Pakistan
}

\begin{abstract}
A 78-year female presented with the complain of per rectal fresh bleeding for 4 days. She was known to have diabetes and hypertension, 3 weeks back. She had an episode of left middle cerebral artery (MCA) stroke. After stroke, she suffered from upper limb weakness and aphasia. At the time of presentation, her vitals showed blood pressure of $118 / 52 \mathrm{mmHg}$, $\mathrm{O}_{2}$ saturation of $98 \%$, temperature: $37^{\circ} \mathrm{C}$, respiratory rate $(\mathrm{RR})$ of $20 / \mathrm{min}$, and heart rate (HR) of $90 \mathrm{bpm}$. After achieving hemodynamic stability, she was transferred to radiology department. Her presenting complain of active rectal bleeding was managed by interventional radiologist using angiographic embolisation. In this patient, it was found pooling of blood in a retrograde fashion in the sigmoid colon. Bleeding was initially believed to be coming from sigmoid arteries seen on images of CT scan and colonoscopy. However, arteriography showed that source of bleeding was from middle and inferior rectal arteries that originated from left internal iliac artery. The intervention radiology (IR) team had to put in extensive effort to locate and perform therapeutic embolisation.
\end{abstract}

Key Words: Therapeutic Embolisation, Angiography, Gastrointestinal hemorrhage.

How to cite this article: Azeemuddin M, Khan MK, Barakzai MD, Khan MGA, Sayani R, Khan DBA. Angiography and therapeutic embolisation of bleeding aberrant lliac artery branches. J Coll Physicians Surg Pak 2020; 30(3):327-329.

\section{INTRODUCTION}

In 2018, the World Health Organization published GLOBOCAN database, according to which colorectal cancer is the third most commonly diagnosed cancer in males and the second in females, with around 1,849,518 new cases and almost 880,792 deaths. ${ }^{1}$ In 2011, Bhurgri et al. reported the crude incidence rate of rectal cancer as 2.3/100,000 in 1998-2002 in Karachi, Pakistan. ${ }^{2}$ In Pakistan, due to absence of functioning national cancer registry, there is no data on current incidence or prevalence of different cancers in the country. However, in 2018 , seven studies were evaluated for determining the prevalence of colorectal cancer, which showed an overall prevalence of $5 \% .^{3}$ Bleeding from rectum is one of the common complains noted in patients presenting to the emergency department. In Pakistan, the common causes of rectal bleeding include hemorrhoids $(22.3 \%)$, inflammatory bowel diseases $(17.1 \%)$, solitary rectal ulcers $(7.4 \%)$, and polyps (14.3\%). ${ }^{4}$ The most common combination of symptoms noted in patients with rectal cancer were altered bowel habits and rectal bleeding. ${ }^{5,6}$

Correspondence to: Dr. Misha Khalid Khan, House No. 9/142, Defence Officers Colony, Thandi Sarak, Ghaznavi Street, Hyderabad, Pakistan

E-mail:mishakhalid.k@gmail.com

Received: December 17, 2018; Revised: July 05, 2019; Accepted: August 22, 2019
Hence, it is important to thoroughly evaluate the cause of gastrointestinal tract bleed.

\section{CASE REPORT}

A 78-year female, came to the Emergency Department with the complain of active per rectal bleeding and drowsiness for 4 days. She was known to have hypertension and diabetes. Around 25 days ago, she developed right upper limb weakness and aphasia secondary to left middle cerebral artery (MCA) infarction. After this infarct, she was started on anti-platelet drugs (aspirin \& transamine). These medications were prescribed to her from other hospitals. At the time of presentation to our hospital, her vitals showed blood pressure of $118 / 52 \mathrm{mmHg}, \mathrm{O}_{2}$ saturation of $98 \%$, temperature $37^{\circ} \mathrm{C}$, respiration rate (RR) $20 / \mathrm{min}$ and pulse of $90 \mathrm{bpm}$. Patient did not report any chest pain, palpitations, and her electrocardiography (ECG) and troponin levels were normal. She was drowsy and was actively bleeding fresh blood with clots. She was admitted for further workup and management.

Due to active bleeding, patient's initial hemoglobin $(\mathrm{Hb})$ dropped from 9 to $6 \mathrm{~g} / \mathrm{dL}$ with hematocrit (Hct) of $19.9 \%$. Colonoscopy revealed large internal hemorrhoids and multiple rectal ulcers of different sizes. Active bleeding was seen, and blood collection was present in sigmoid colon. Bleeding was suspected to be coming 


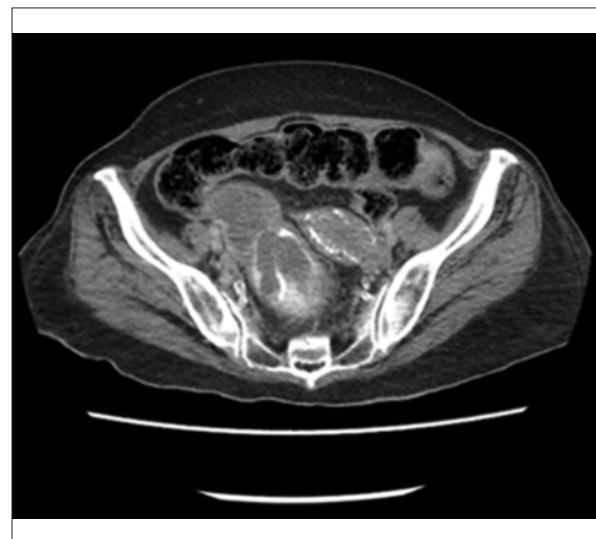

Figure 1: Contrast enhanced CT (axial section), showing high density material in the sigmoid colon and rectum (black arrow), representing active (bleeding) extra-vasation and accumulation of contrast.

from sigmoidal arteries. Biopsy was taken from these lesions. Biopsy report stated that rectum was severely inflamed; and ulcerated colonic mucosa showed viral inclusion bodies suggestive of cytomegalovirus infection.

For her drowsiness, MRI brain with magnetic resonance angiography (MRA) was done, which revealed acute infarct of left MCA perforators. Cranial time-of-flight (TOF) MRA showed severe intracranial atherosclerotic changes with multiple areas of high-grade stenosis. Due to severe intracranial atherosclerotic changes, she ideally needed dual antiplatelets; and anticoagulation but after her colonoscopy findings, she was considered a poor candidate for anticoagulation. Thus, it was decided to start one anti-platelet agent after one week in clinic.

After achieving hemodynamic stability, she was transferred to radiology department. CT scan of abdomen on unenhanced view showed high density material in sigmoid colon and rectum. CT with contrast showed accumulation of contrast in sigmoid colon. Delayed phase showed active bleeding (Figure 1). Colonoscopy and CT scan results showed that the source of bleeding was from branches of sigmoid arteries.

Interventional radiology (IR) team was consulted. Patient was taken to the interventional suite for a mesenteric angiography. After standard aseptic measures and anesthesia, right femoral artery was punctured with needle; and using Seldinger's technique, 4 Fr vascular access sheath was placed followed by insertion of $4 \mathrm{Fr}$ SIM 11 catheter. Selective catheterisation of superior mesenteric artery (SMA); and inferior mesenteric artery (IFA) did not show any active extravasation (Figure 2). Extensive superselective catheterisation was done to locate the bleeder. Right internal iliac artery also did not show any active bleeding. Finally, selective catheterisation of left internal iliac artery revealed active bleeding from middle and inferior rectal branches (Figure 3). This profuse bleeding was going in a retrograde direction due to peristalsis and had collected in sigmoid colon.
CT and colonoscopy gave a false picture of active bleeding coming from sigmoid colon. However, after arteriography, it was concluded that the actual source of bleeding was from left Internal Iliac artery branches. Embolisation was performed after super-selectively cannulating these branches using microcatheter and 250-355 poly-vinyl-alcohol (PVA) particles followed by deployment of coils $(2 \times 2 \mathrm{~mm})$. No immediate postprocedural complications were noted. After the procedure, the patient remained vitally stable, active and alert without any active complains. At the time of discharge, her $\mathrm{Hb}$ was $13.3 \mathrm{~g} / \mathrm{dL}$ with Hct $39.5 \%$.

\section{DISCUSSION}

Abdominal aorta, before its bifurcation, gives off inferior mesenteric artery (IMA), that terminates inferiorly as the superior rectal artery (SRA). Sigmoid colon is most commonly supplied by sigmoid arteries coming from IMA. The internal iliac arteries, gives off middle rectal artery (MRA), which supplies blood to distal rectum and proximal anal canal. The presence of these arteries is variable. According to Havaldar et al., the course of MRA is variable in terms of its origin and caliber. In their population, it was present in only $72 \%$ of the subjects observed. The MRA was seen to arise most frequently as a branch of the internal pudendal artery in $54 \%$ of the observed cases. ${ }^{7}$ The inferior rectal arteries (IRA) arise from the internal pudendal artery, which is a branch of the internal iliac artery. Low incidence of rectal ischemia is due to presence of intramural collaterals between the superior and inferior rectal arteries. ${ }^{7}$

Previously, few patients from South Asia had third anomalous branch, named as accessory left colic artery (ALCA). One such patient had ALCA branching off from the IMA and further dividing into two transverse branches taking part in the formation of arterial arc for the perfusion of the transverse colon (TC) and the spleenic flexure. Both these branches of the ALCA 
formed arterial arcade for the large bowel and supplied the left one-third of the TC and the proximal part of the descending colon. 8

First use of diagnostic mesenteric angiography was reported in 1963. Today, CT angiography (CTA) is an effective modality used for diagnostic and therapeutic purposes, in patients with continuous bleeding. Studies have shown that CTA has sensitivity of $92-100 \%$ in localising active lower gastrointestinal bleeding. Other methods of detection include wireless capsule endoscopy, double-balloon enteroscopy and radionuclide Meckel's scan. Diagnostic yield for double balloon enteroscopy and capsule endoscopy is reported to be $55-65 \%$. However, there have been incidences when lesions were missed by capsule endoscopy and later diagnosed using double balloon enteroscopy. ${ }^{9}$

After detection of active bleeding area, we can proceed with therapeutic intervention by either vasopressin infusion or embolisation. Vasopressin will stop bleeding in $90 \%$ of patients. It causes decreased blood supply by vasoconstriction. However, after cessation of vasopressin infusion, incidence of rebleeding were seen in $50 \%$ cases. Hence, it has been categorised as secondline treatment after embolisation. ${ }^{9}$ Endovascular angiography is a diagnostic and therapeutic tool that can be done in emergency cases without any bowel preparation. Using superselective transarterial embolisation, our aim was to reduce arterial perfusion pressure and main adequate collateral supply. 10 Transcatheter embolisation is now recommended as an effective and safe procedure. There are very few adverse events like mucosal ischemia and stricture formation that have been reported. Superselective embolisation is now recommended as the first angiographic therapy for lower gastrointestinal bleeding. 9 Previous studies do not mention exact success rate from each bleeding rectal vessel. However, clinical success rate of embolisation from upper gastrointestinal bleeding and lower gastrointestinal bleeding is reported to be $44-100 \%$ and 88 $93 \%$, respectively. Furthermore, embolisation of the subsegmental peripheral arterial branches have success rates ranging from $80-100 \%$ with low recurrence rate ranging from $14-29 \% .^{9}$

By this case report, a difficult case was presented that was timely and efficiently managed using superselective catheter embolisation technique. Based on the radiographic findings of this patient, it is concluded that imaging like CT and colonoscopy may sometimes present a false image which can differ from the true result seen on arteriography. Hence, it is imperative for the surgeons and radiologists to look for variations in colonic vasculature to avoid unwanted complications.

\section{PATIENT'S CONSENT:}

Patient's consent was obtained. Patient confidentiality and anonymity was maintained at each step of this process..

\section{CONFLICT OF INTEREST:}

Authors declared no conflict of interest.

\section{AUTHORS' CONTRIBUTION:}

MA: Supervised and critically revised the study.

MKK, MGAK: Conducted review of the current literature and previously reported cases. The case report was written by Khan MK.

MDB, MGAK, RS, DBAK: The findings were critically analysed and evaluated.

All authors read and approved the final version submitted for publication.

\section{REFERENCES}

1. Ferlay J, Colombet M, Soerjomataram I, Mathers C, Parkin DM, Piñeros $\mathrm{M}$, et al. Estimating the global cancer incidence and mortality in 2018: GLOBOCAN sources and methods. Int J Cancer 2019; 144:1941-53.

2. Bhurgri Y, Khan T, Kayani N, Ahmad R, Usman A, Bhurgri A, et al. Incidence and current trends of colorectal malignancies in an unscreened, low risk Pakistan population. Asian Pac J Cancer Prev 2011; 12:703-8.

3. Idrees R, Fatima S, Abdul-Ghafar J, Raheem A, Ahmad Z. Cancer prevalence in Pakistan: Meta-analysis of various published studies to determine variation in cancer figures resulting from marked population heterogeneity in different parts of the country. World J Surg Oncol 2018; 16:129.

4. Jehangiri AR, Gul R, Hadayat R, Khan AN, Zabiullah, Khursheed L. Causes of lower gastrointestinal bleeding on colonoscopy. J Ayub Med Coll Abbottabad 2017; 29:468-71.

5. Adelstein BA, Macaskill P, Chan SF, Katelaris PH, Irwig L. Most bowel cancer symptoms do not indicate colorectal cancer and polyps: a systematic review. BMC Gastroenterol 2011; 11:65.

6. Malik AI, Badar F, Syed AA, Yusuf MA. Surgically treated rectal cancer patients - outcomes at a tertiary care cancer hospital in Pakistan. Asian J Surg 2015; 38:13-20.

7. Havaldar PP, Taz S, Angadi AV, Saheb SH. Study of variations in anterior division of internal iliac artery. Int J Anat Res 2014; 2:363-8.

8. Hansdak R, Pakhiddey R, Thakur A, Mehta V, Rath G. Anatomical description and clinical relevance of a rare variation in the mesenteric arterial arcade. J Clin Diagn Res 2015; 9: AD01-AD02.

9. Raphaeli T, Menon R. Current treatment of lower gastrointestinal hemorrhage. Clin Colon Rectal Surg 2012; 25:219-27.

10. Ramaswamy RS, Choi HW, Mouser HC, Narsinh KH, McCammack KC, Treesit $\mathrm{T}$, et al. Role of interventional radiology in the management of acute gastrointestinal bleeding. World J Radiol 2014; 6:82-92. 